\title{
The influence of essential fatty acids on the female health
}

\author{
Ceci Mendes Carvalho Lopes ${ }^{\top} \odot$, Lucia de Fatima Cahino da Costa Hime ${ }^{1} \odot$, \\ Edmund Chada Baracat ${ }^{\top} \odot$, Jose Maria Soares-Júnior ${ }^{1 *} \odot$
}

\section{INTRODUCTION}

Essential fatty acids are polyunsaturated fatty acids (PUFAs) that are not synthesized in the organisms. Therefore, PUFAs are needed to be supplied through the diet for proper functioning of the metabolic processes. There are several sources of PUFAs, such as some oils (soybeans and canola), some seeds (pumpkin, chia, and sunflower), leafy vegetables, nuts, fish, and shellfish ${ }^{1}$. The essentiality of these acids was defined in 1930, when rats fed with a deficiency of PUFAs developed dermatological, renal, and visual problems and fertility disorders, and that the reintroduction of these acids reversed the symptoms ${ }^{1-3}$. PUFAs are considered essential because they are the precursors of important substances, such as prostaglandins (PGs), thromboxanes (TXs), and leukotrienes (LTs), denominate eicosanoids ${ }^{2}$, and act in various functions of the organisms.

The nutritional supply is generally ample, and PUFAs may find in various types of food. Basically, there are two types of PUFAs, namely, omega- 3 and omega- 6 , defined by the location of the first double bond in the initial molecule of the carbon atom in relation to the methyl end of the chain ${ }^{2,3}$. The three types of omega-3 fatty acids involved in human physiology are alpha-linolenic acid (ALA), found in plant oils, eicosapentaenoic acid (EPA), and docosahexaenoic acid (DHA). Linolenic acid (LA), the shortest chain of the common omega- 6 fatty acids in the human diet, is categorized as an essential fatty acid because the human body cannot synthesize it ${ }^{2,3}$.

The omega- 6 fatty acids are precursors to endocannabinoids, lipoxins, and specific eicosanoids. LA converts to gamma-linolenic acid (GLA), while GLA converts to EPA. GLA is a precursor to prostaglandins and leukotrienes. EPA is a precursor of anti-inflammatory factors (lipoxins, resolvins, and protectins $)^{3,4}$. In some situations, such as aging, insulin resistance, autoimmune diseases, obesity, a diet with a predominance of saturated fats, and alcohol intake, there is a tendency for the activity of enzymes involved in PUFA metabolism to decrease, requiring the introduction of supplementary diet consumption'

The balance between the intake of omega- 3 and omega- 6 fatty acids prevents the appearance of chronic inflammatory diseases, cardiovascular diseases, and some cancers. The "Western diet" has more LA than ALA. There are controversies about the ideal ratio, but it is considered ideal when the ratio between omega- 3 and omega- 6 is $1: 4$, respectively, to avoid the risk of developing cardiovascular diseases ${ }^{6}$.

\section{The action of polyunsaturated fatty acids on immunity}

Although immunity and resistance to infection are dependent on several factors, and therefore very different between people, nutrition can significantly influence it not only as a factor but also as interacting with others (genetics, environment, and lifestyle). Among the nutrients, there is a prominent place for essential fatty acids ${ }^{6,7}$.

Omega-3s have been well studied and shown to play a significant role in cellular immune function. Omega-6s have not seemed to perform that preponderantly. In many studies, they are used as controls for omega-3s. This potent activity of omega-3s is due to their high capacity to inhibit the production of inflammatory mediators. Among these eicosanoids, everything indicates that they also participate in a process of gene activation, prostaglandin E2, and some leukotrienes. There are also pro-inflammatory cytokines, adhesive molecules, platelet-activating factors, and reactive oxygen and nitrogen species ${ }^{7}$.

An extensive and detailed review addresses the metabolic and genetic mechanisms of PUFA action on immunity and inflammation. They demonstrate that this process is, in many respects,

\footnotetext{
'Universidade de São Paulo, Hospital das Clínicas, Faculdade de Medicina, Departamento de Obstetrícia e Ginecologia - São Paulo (SP), Brazil. *Corresponding author: jsoares415@hotmail.com

Conflicts of interest: the authors declare there are no conflicts of interest. Funding: none.

Received on March 20, 2021. Accepted on August 13, 2021.
} 
quite paradoxical, as, in similar situations, it sometimes leads to even opposite effects. The conclusion they reach is that there are many individual variables, both metabolic and even genetic, and that, therefore, there is no way to establish a dose or a therapeutic scheme with them, as this has to be established individually ${ }^{8}$.

\section{Asthma and allergic rhinitis}

Based on the prior knowledge, leukotrienes are very important in triggering asthma. In fact, leukotrienes come from the metabolism of arachidonic acid (AA), via the 5-lipoxygenase pathway, and that they also have numerous pro-inflammatory properties, which also significantly contribute to the onset of asthma and allergic rhinitis. Some authors decided to study the activity of a mixture of borage oil and echium oil, i.e., another borage, which are rich in omega- 6 fatty acids (and therefore do not produce AA in their metabolic cascade) and its impact on the inflammatory mechanism? ${ }^{9}$. Specifically, they selected 37 asthmatic participants and divided them into four groups. Each group received capsules of a mixture of oil from the two plants (1,000 $\mathrm{mg}$ of echium and 1,300 $\mathrm{mg}$ of borage), respectively, in increasing doses for each group, for three weeks. The authors found a decrease in the PUFA levels and an attenuation of the production of leukotrienes in patients with asthma?.

Several studies since the 1980s have shown that oils rich in GLA can promote improvements in various inflammatory diseases, such as asthma, atopic dermatitis, and rheumatoid arthritis. Some of the studies evaluated the combination of omega- 6 and omega-3 and demonstrated a reduction in cytokines and leukocyte contribution, including in patients with severe lung injury, requiring intensive care. However, these studies did not have uniformity, and the number of participants was low. Therefore, further studies are necessary for a consistent framework of conclusions ${ }^{8}$.

Studies show that populations who use the "Mediterranean diet" present fewer cases of asthma than those who eat the "Western diet." The Mediterranean diet, rich in vegetables and fish, is a great source of essential fatty acids. The "Western diet" tends to be pro-inflammatory. In addition, this diet favors obesity, another triggering factor for asthma ${ }^{10}$.

\section{Rheumatoid arthritis}

Rheumatoid arthritis occupies a prominent place among autoimmune diseases. Pathogenesis is associated with the inflammatory agents that infiltrate the joints, producing swelling and pain. Several studies have proven the action of omega-3 fatty acids in improving the clinical picture of this disease. Some omega-6s exert this benefit, especially GLA, found in borage, evening primrose, and black currant, because this substance may be converted to dihomo-gamma-linolenic acid (DHLA), which has a potent anti-inflammatory ${ }^{11}$.
A double-blind study was carried out dividing 60 patients into three groups of 20 , in which all participants received the recommended traditional treatment. The first group of patients also received a daily postprandial dose of five fish oil capsules (300 mg of DHA, $200 \mathrm{mg}$ EPA, and $100 \mathrm{mg}$ of other PUFAs); the second group received a daily postprandial dose double capsules of the first group and two capsules of evening primrose oil (each containing 1,300 mg-949 mg LA and $117 \mathrm{mg} \mathrm{GLA}$ ); and the third group received only placebo. Clinical improvement was observed in both the first and second groups ${ }^{11}$.

\section{Action on cardiovascular health}

\section{Myocardial infarction and stroke}

About one-third of deaths around the world may be attributed to atherosclerosis that causes cardiovascular disease and stroke ${ }^{12}$.

PUFAs are the potent inhibitors of 3-hydroxy-3-methylglutaryl coenzyme A (HMG-CoA) reductase that is essential in cholesterol metabolism. Statins act on this enzyme, known as potent lipidemia reducers, so significantly involved in atherosclerosis and, consequently, in infarction and strokes. There are strong indications that PUFAs act as mediators of statin action. If administered together, as a medicine, they can potentiate its action on the protection. PUFAs act as antiplatelet agents which is considered an important action in both the treatment and prevention of myocardial infarction ${ }^{13}$.

Based on the knowledge that PUFAs, especially omega-3s, play a major role in atherosclerosis, although omega-6s are considered antagonists (due to their pro-inflammatory action), some omega-6s also play a beneficial role. Significant in this process, a group of British and Israeli researchers sought to investigate the mechanisms. Their studies led to the determination of several cellular mechanisms. In addition, the authors demonstrated that DHLA, derived from GLA, may play an important role in this process ${ }^{12,13}$.

DHLA has an antithrombotic activity, which is important for decreasing arterial thrombotic events that lead to circulatory obstruction and serious damage. Although statins promote an obvious improvement in the prognosis of cardiovascular, they present side effects, which decrease the treatment adherence. PUFAs have been referred to as having great therapeutic and adjuvant possibilities for a long period of treatment. As inflammation is closely linked to the action of cytokines, in triggering atherosclerosis, DHLA has been proven to decrease the release of these cytokines. However, further studies are necessary ${ }^{12}$.

\section{Systemic arterial hypertension}

Following the data recorded in the National Health and Nutrition Examination Survey (NHANES) 2007-2014, the adequate 
balance between omega- 6 and omega- 3 intake is associated with a lower incidence of hypertension ${ }^{14}$.

A study carried out with postmenopausal hypertensive patients with $2 \mathrm{~g}$ of GLA per day for six months proves the effect on blood pressure without the appearance of side effects ${ }^{15}$.

PUFAs influence the action of the renin-angiotensin system, acting in the reduction of hypertensive processes. Its action is known to improve renal plasma flow, reduce renal vascular resistance, and favor glomerular filtration. This effect may act protectively and preserve kidney function ${ }^{13-17}$.

\section{Dry eye syndrome}

Dry eye is a problem that can interfere a lot in people's daily lives, especially affecting activities that require the use of vision, such as reading, driving at night, using the computer, and so on, both professionally and for dilettantism. Some factors seem to influence its appearance, such as aging, female gender, inflammatory and immune processes as well as hypoestrogenism and hyperprolactinemia ${ }^{17,18}$.

PUFAs, especially omega- 3 and omega- 6 , seem to ameliorate the prevalence of dry eye. In fact, the authors suggested that effect after systematic review ${ }^{1}$. After three generations of rats were fed with the omega-3 deficit, there was no difference in the severity of the condition (dry eye) in the group with and without deficiency. However, several clinical studies, using both topically and by ingestion, diets or supplements balanced with omega- 3 and omega- 6 have shown an improvement in the syndrome. The explanation lies in the anti-inflammatory action of these fatty acids and leads to the obvious suggestion of prescribing them to the affected people. However, further study is necessary for adjusting the dose for the routine therapeutic protocol ${ }^{1}$.

\section{Obesity and colorectal cancer}

It is well known that obesity is an inflammatory disease and plays an important role in the development of colorectal cancer. In fact, adipocytes release immunosuppressive signals, associated with a pro-inflammatory state, and omega-6s seem to be of importance in this process. The nutritional diet with the imbalance between omega- 3 and omega- 6 may participate in the pathogenesis of colorectal cancer, although increasing inflammation, acting on lymphocytes, and interfering with the release of cytokines and chemokines ${ }^{16}$.

\section{Action in menopause}

Although the use of essential fatty acids is not usually highlighted among the various treatment options, some authors have administered them to climacteric patients, including studies in Brazil. Although, in some of them, there was no difference between treated cases and controls, some described benefits such as improved well-being and quality of life, improvement in symptoms such as memory capacity and depressive states and irritability, and even symptoms, i.e., vasomotor, with the use of omega- 6 capsules with ${ }^{3} 2 \mathrm{~g}$ per day. Improvements were also found in processes associated with the climacteric patients, such as high blood pressure and even the distribution of body fat (a risk factor for heart disease and the leading cause of lethality worldwide) $)^{15,17}$.

\section{Action during pregnancy}

Discussing the importance of the Mediterranean diet, rich in vegetables and fish, that this diet therefore promotes the administration of essential fatty acids and that people who are nourished with this type of food are less prone to asthma, it is also reported that the administration of fatty acids, such as GLA, during pregnancy promotes the birth of children with a lower incidence of allergic problems, such as asthma ${ }^{10}$.

A Cochrane review addressing studies with the administration of omega-3 supplements during pregnancy (covering a total of almost 20,000 patients) suggested that these substances may reduce the preterm births, despite a certain increase in prolonged pregnancies beyond 42 weeks, which increased the need for interventions. The authors suggest that therefore the administration of supplements with omega-3 to pregnant women may be an option for pregnancy ${ }^{19}$. They also confirm the beneficial action of DHA during pregnancy and breastfeeding for at least 6 months with a positive response in cognitive abilities ${ }^{20}$.

\section{CONCLUSION}

PUFAs participate in several metabolic processes acting not only in the prevention but also in the treatment of several diseases. Corroborating the aforementioned authors, we indicate a nutritional diet with PUFAs for improving female health.

\section{REFERENCES}

1. Al Mahmood AM, Al-Swailem SA. Essential fatty acids in the treatment of dry eye syndrome: a myth or reality? Saudi J Ophthalmol. 2014;28(3):195-7. https://doi.org/10.1016/j. sjopt.2014.06.004
2. Stone KJ, Willis AL, Hart WM, Kirtland SJ, Kernoff PB, McNicol GP. The metabolism of dihomo-gamma-linolenic acid in man. Lipids. 1979;14(2):174-80. https://doi.org/10.1007/ BF02533869 
3. Wills AL, Smith DL. New protective roles for selectd nutrientes. Dihommo-gamma-linolenic and gamma-linolenic acids in health diseases. New York: Spiller GA, Scala editors; 1989. p.39-108.

4. Lopes CMC, Lima SMRR, Veiga ECA, Soares-Júnior JM, Baracat EC. Phytotherapeutic medicines: reality or myth? Rev Assoc Med Bras (1992). 2019;65(3):292-4. https://doi. org/10.1590/1806-9282.65.3.292

5. Czumaj A, Śledziński T. Biological role of unsaturated fatty acid desaturases in health and disease. Nutrients. 2020;12(2):356. https://doi.org/10.3390/nu12020356

6. Mariamenatu AH, Abdu EM. Overconsumption of omega- 6 polyunsaturated fatty acids (pufas) versus deficiency of omega-3 pufas in modern-day diets: the disturbing factor for their "balanced antagonistic metabolic functions" in the human body. J Lipids. 2021;2021:8848161. https://doi. org/10.1155/2021/8848161

7. Wu D, Lewis ED, Pae M, Meydani SN. Nutritional modulation of immune function: analysis of evidence, mechanisms, and clinical relevance. Front Immunol. 2019;9:3160. https://doi. org/10.3389/fimmu.2018.03160

8. Sergeant S, Rahbar E, Chilton FH. Gamma-linolenic acid, Dihommo-gamma linolenic, eicosanoids and inflammatory processes. Eur J Pharmacol. 2016;785:77-86. https://doi. org/10.1016/j.ejphar.2016.04.020

9. Arm JP, Boyce JA, Wang L, Chhay H, Zahid M, Patil V, et al. Impact of botanical oils on polyunsaturated fatty acid metabolism and leukotriene generation in mild asthmatics. Lipids Health Dis. 2013;12:141. https://doi.org/10.1186/1476-511X-12-141

10. Guilleminault L, Williams EJ, Scott HA, Berthon BS, Jensen M, Wood LG. Diet and asthma: is it time to adapt our message? Nutrients. 2017;9(11):1227. https://doi.org/10.3390/ nu9111227

11. Veselinovic M, Vasiljevic D, Vucic V, Arsic A, Petrovic S, TomicLucic $A$, et al. Clinical benefits of n-3 PUFA and $r$-Linolenic acid in patients with rheumatoid arthritis. Nutrients. 2017;9(4):325. https://doi.org/10.3390/nu9040325

12. Gallagher $\mathrm{H}$, Williams JO, Ferekidis $\mathrm{N}$, Ismail A, Chan $\mathrm{YH}$, Michael DR, et al. Dihomo- $\gamma$-linolenic acid inhibits several key cellular processes associated with atherosclerosis. Biochim
Biophys Acta Mol Basis Dis. 2019;1865(9):2538-50. https:// doi.org/10.1016/j.bbadis.2019.06.011

13. Das UN. Essential fatty acids and their metabolites could function as endogenous HMG-CoA reductase and ACE enzyme inhibitors, anti-arrhythmic, anti-hypertensive, anti-atherosclerotic, antiinflammatory, cytoprotective, and cardioprotective molecules. Lipids Health Dis. 2008;7:37. https://doi.org/10.1186/1476-511X-7-37

14. Chen J, Sun B, Zhang D. Association of dietary $n 3$ and $n 6$ fatty acids intake with hypertension: NHANES 2007-2014. Nutrients. 2019;11(6):1232. https://doi.org/10.3390/nu11061232

15. Hime LFCC, Lopes CMC, Roa CL, Zuchelo LTS, Baracat EC, Andrade J, et al. Is there a beneficial effect of gamma-linolenic acid supplementation on body fat in postmenopausal hypertensive women? A prospective randomized double-blind placebocontrolled trial. Menopause. Menopause. 2021;28(6):699-705. https://doi.org/10.1097/GME.0000000000001740

16. Del Cornò $M, D^{\prime}$ Archivio $M$, Conti $L$, Scazzocchio $B$, Varì $R$, Donninelli $G$, et al. Visceral fat adipocytes from obese and colorectal cancer subjects exhibit distinct secretory and $\omega 6$ polyunsaturated fatty acid profiles and deliver immunosuppressive signals to innate immunity cells. Oncotarget. 2016;7(39):63093-105. https://doi.org/10.18632/ oncotarget.10998

17. Hime LFCC, Lopes CMC. Climatério: ômega 6 e suas propriedades terapêuticas. In: Lima SMRR. Fitomedicamentos na prática ginecológica e obstétrica. 2nd ed. São Paulo: Editora Atheneu; 2009. p.293-300.

18. Verna C, Soares JM, Martins FW, Teixeira RC, Mosquette $R$, Simões RS, et al. Efeito da hiperprolactinemia induzida pela metoclopramida na córnea de camundongas. Arq Bras Oftalmol. 2006;69(5):645-9. https://doi.org/10.1590/s000427492006000500004

19. Middleton P, Gomersall JC, Gould JF, Shepherd E, Olsen SF, Makrides M. Omega-3 fatty acid addition during pregnancy. Cochrane Database Syst Rev. 2018;11(11):CD003402. https:// doi.org/10.1002/14651858.CD003402.pub3

20. Mallick R, Basak S, Duttaroy AK. Docosahexaenoic acid,22:6n-3: Its roles in the structure and function of the brain. Int J Dev Neurosci. 2019;79:21-31. https://doi.org/10.1016/j. ijdevneu.2019.10.004 